\title{
Erratum to: Percolation on random Johnson-Mehl tessellations and related models
}

\author{
Béla Bollobás · Oliver Riordan
}

Published online: 31 October 2009

(C) Springer-Verlag 2009

\section{Erratum to: Probab. Theory Relat. Fields (2008) 140:319-343 DOI 10.1007/s00440-007-0066-1}

The proof presented in [2] of the result that the critical probability for percolation on a random Johnson-Mehl tessellation is $1 / 2$ contains an obvious error; we are very grateful to Rob van den Berg for bringing this to our attention. Fortunately, this error is easy to correct. There is a class of influence results, especially those of Talagrand [9] and Friedgut and Kalai [5] (in both cases extending results of Kahn, Kalai and Linial [6] and Bourgain, Kahn, Kalai, Katznelson and Linial [4]), whose application to prove sharp thresholds for 'symmetric' events works particularly well. As so often in such settings, to prove that a threshold is sharp, one needs only enough symmetry to ensure that many variables are equivalent, rather than total symmetry; the latter is not present in [2].

The online version of the original article can be found under doi:10.1007/s00440-007-0066-1.

B. Bollobás research was supported in part by NSF grants DMS-0906634, CNS-0721983 and CCF-0728928, and ARO grant W911NF-06-1-0076.

\section{B. Bollobás}

Department of Pure Mathematics and Mathematical Statistics,

University of Cambridge, Wilberforce Road, Cambridge CB3 0WB, UK

B. Bollobás

Department of Mathematical Sciences, University of Memphis,

Memphis, TN 38152, USA

O. Riordan $(\varangle)$

Mathematical Institute, University of Oxford,

24-29 St Giles', Oxford OX1 3LB, UK

e-mail: riordan@maths.ox.ac.uk 
There are many applications of these and other influence results which do not require any symmetry, starting with Russo's proof of Kesten's Theorem [8]; however, such arguments do not seem to apply (at least without extra work) in the context here, where the discrete product probability space is made up of a very large number of variables each of which has only a very small probability of being non-zero, and we require the width of the threshold to be small in terms of the relative changes in these probabilities. This situation arises naturally when discretizing continuous processes such as Poisson processes.

Let $\mathbb{P}_{p_{-}, p_{+}}^{n}$ denote the probability measure on $\{-1,0,1\}^{n}$ in which each coordinate is independent, and is equal to +1 with probability $p_{+}$, and to -1 with probability $p_{-}$. An event $E \subset\{-1,0,1\}^{n}$ is increasing if whenever $x \in E$ and the inequality $x \leq x^{\prime}$ holds coordinatewise, then $x^{\prime} \in E$. We say that $E$ has symmetry of order $m$ if there is a group action on $[n]=\{1,2, \ldots, n\}$ in which each orbit has size at least $m$, such that the induced action on $\{-1,0,1\}^{n}$ preserves $E$. To correct the proof in [2] we need the following lemma.

Lemma 1 There is an absolute constant $c_{3}>0$ such that if $0<q_{-}<p_{-}<1 / e$, $0<p_{+}<q_{+}<1 / e, E \subset\{-1,0,1\}^{n}$ is increasing and has symmetry of order $m$, and $\mathbb{P}_{p_{-}, p_{+}}^{n}(E)>\eta$, then $\mathbb{P}_{q_{-}, q_{+}}^{n}(E)>1-\eta$ whenever

$$
\min \left\{q_{+}-p_{+}, p_{-}-q_{-}\right\} \geq c_{3} \log (1 / \eta) p_{\max } \log \left(1 / p_{\max }\right) / \log m,
$$

where $p_{\max }=\max \left\{q_{+}, p_{-}\right\}$.

Using this result in place of Theorem 2.2 of [1] (which is simply the special case when $m=n$, i.e., when $E$ is symmetric), the proof in [2] may be corrected with essentially no changes. Indeed, the event $E_{3}$ considered at the bottom of page 329 , that some $3 s / 4$ by $s / 12$ rectangle in $\mathbb{T}(s)$ has a robustly black horizontal crossing, is symmetric under translations of the space $\mathbb{T}(s) \times[0, s]$ in which the Poisson point processes live through vectors of the form $(x, y, 0)$. Hence the corresponding discrete event $E_{3}^{\text {crude }}$ considered on the next page has symmetry of order $m=(s / \delta)^{2}$. To deduce Theorem 6 of [2] from Lemma 1 one needs the inequality in the middle of page 330 of [2], but with $\log N$ replaced by $\log m$. Since $N=(s / \delta)^{3}$, this corresponds simply to a change in the constant, and all remaining calculations are unaffected.

To prove Lemma 1 one needs a suitable influence result. Such a result was proved for a product of 2-element spaces by Talagrand [9]; later, Friedgut and Kalai [5] used a different method to obtain slightly weaker results. One can adapt Talagrand's proof to the 3-element setting, obtaining a slightly weaker form of Lemma 1 (see the remark at the end of the full version [3] of this note), but it seems easier to follow the method of [5]. Unfortunately, even in the two element case, Friedgut and Kalai did not prove the precise result we need, although their method gives it.

Given a function $f$ on a product probability space $\Omega^{n}$, let $I_{f}(k)$ denote the influence of the $k$ th coordinate with respect to $f$, i.e., the probability of the set of configurations $\omega$ with the property that there is some $\omega^{\prime}$ differing from $\omega$ only in the $k$ th coordinate for which $f\left(\omega^{\prime}\right) \neq f(\omega)$. For $A \subset \Omega^{n}$, let $I_{A}(k)=I_{f}(k)$ where $f$ is the characteristic function of $A$. 
Following the notation of Friedgut and Kalai [5], let $V_{n}(p)$ denote the weighted cube, that is the $n$th power of the 2-element probability space in which $\mathbb{P}(0)=1-p$ and $\mathbb{P}(1)=p$. Bourgain, Kahn, Kalai, Katznelson and Linial [4] showed that if $f$ is any $0 / 1$-valued function on the $n$th power of a 'standard' probability space (such as a discrete space, or the interval $[0,1])$, then some influence $I_{f}(k)$ is at least a constant times $t \log n / n$, where $t=\min \left\{\mathbb{P}\left(f^{-1}(0)\right), \mathbb{P}\left(f^{-1}(1)\right)\right\}$. Friedgut and Kalai [5] adapted their proof to prove two extensions (Theorems 3.1 and Theorem 3.4 in [5]). Although they did not do so, there is no problem combining these two extensions, to obtain the following result; for details see [3].

Lemma 2 Let $0<p \leq 1 / 2$ and let $f: V_{n}(p) \rightarrow\{0,1\}$ with $\mathbb{P}\left(f^{-1}(1)\right)=t$. If $I_{f}(k) \leq \delta$ for every $k$ then $I_{f}=\sum_{k=1}^{n} I_{f}(k)$ satisfies the inequality

$$
I_{f} \geq c \frac{t(1-t)}{p \log (1 / p)} \log \left(\frac{c t(1-t)}{\delta^{1 / 2} I_{f}}\right),
$$

where $c>0$ is an absolute constant. In particular, if for some $a \leq 1 / 2$ we have $I_{f}(k) \leq a p^{2} \log (1 / p)^{2}$ for every $k$, then

$$
I_{f} \geq c^{\prime} \frac{t(1-t)}{p \log (1 / p)} \log (1 / a),
$$

where $c^{\prime}>0$ is an absolute constant.

Lemma 2 is also implied by Corollary 1.2 of Talagrand [9] (see [3]). However, a key feature of the approach in [5] is that, as in [4], the first step is to replace each factor $V_{1}(p)$ in the product space $V_{n}(p)$ by the probability space $Y=\{0,1\}^{m}$ with uniform measure; one can assume that $p$ is a dyadic rational, choose $m$ so that $2^{m} p$ is an integer, and take the first $(1-p) 2^{m}$ points of $Y$ (in the binary order) to correspond to $0 \in V_{1}(p)$ and the last $p 2^{m}$ to $1 \in V_{1}(p)$. Then, as noted in [5], for any function $f: V_{1}(p) \rightarrow\{0,1\}$, the sum $w(f)$ of the influences of the corresponding function on $Y$ satisfies

$$
w(f) \leq c_{1} p \log (1 / p)
$$

for some absolute constant $c_{1}$. As in [1], this implies that the extension of Lemma 2 to the probability space $W_{p_{-}, p_{+}}^{n}$, i.e., $\{-1,0,1\}^{n}$ with the product measure $\mathbb{P}_{p_{-}, p_{+}}^{n}$ (with $\left.p_{-}, p_{+} \leq 1 / e\right)$, is immediate. Indeed, replacing each factor $W_{p_{-}, p_{+}}$by $Y=\{0,1\}^{m}$, one now has

$$
w(f) \leq c p_{+} \log \left(1 / p_{+}\right)+c p_{-} \log \left(1 / p_{-}\right) \leq 2 c p_{\max } \log \left(1 / p_{\max }\right)
$$

in place of (2). From this point on the original probability space is irrelevant, and one obtains the following result (see [3] for details). 
Corollary 3 For every $0<p_{-}, p_{+} \leq 1 /$ e and every function $f: W_{p_{-}, p_{+}}^{n} \rightarrow\{0,1\}$ with $\mathbb{P}\left(f^{-1}(1)\right)=t$, if $a \leq 1 / 2$ and $I_{f}(k) \leq a p_{\max }^{2} \log \left(1 / p_{\max }\right)^{2}$ for every $k$, then

$$
I_{f} \geq c \frac{t(1-t)}{p_{\max } \log \left(1 / p_{\max }\right)} \log (1 / a),
$$

where $p_{\max }=\max \left\{p_{-}, p_{+}\right\}$and $c>0$ is an absolute constant.

Corollary 3 easily implies Lemma 1.

Proof of Lemma 1 Since the left-hand side of (1) is at most $p_{\max }$, taking $c_{3}$ large we may assume that $\log m \geq 100 \log \left(1 / p_{\max }\right)$, say, i.e., that $m \geq p_{\max }^{-100}$.

For $0 \leq h \leq h_{\max }=\min \left\{q_{+}-p_{+}, p_{-}-q_{-}\right\}$let $r_{+}=p_{+}+h$ and $r_{-}=p_{-}-h$, and let $g(h)=\mathbb{P}_{r_{-}, r_{+}}^{n}(E)$. Then, by a Margulis-Russo-type formula, the derivative of $g(h)$ is at least $I_{f}=\sum_{k} I_{f}(k)$, where $f$ is the characteristic function of $E$ considered on the product space $\mathbb{P}_{r_{-}, r_{+}}^{n}$. A simple calculation shows that Lemma 1 follows if for every $h$ we have

$$
I_{f} \geq 2 c_{3}^{-1} \frac{t(1-t)}{p_{\max } \log \left(1 / p_{\max }\right)} \log m,
$$

where $t=g(h)=\mathbb{P}_{r_{-}, r_{+}}^{n}(E)$.

To see (3), suppose first that some influence $I_{f}(k)$ is at least $m^{-1 / 2}$, say. Then, from the symmetry assumption, at least $m$ influences are at least this large, and $I_{f} \geq$ $m^{1 / 2} \geq m^{1 / 3} p_{\max }^{-2}$. Taking $c_{3}$ large enough, this is much larger than the bound in (3). (The factor $t(1-t)$ works in our favour.) On the other hand, if $I_{f}(k) \leq m^{-1 / 2}$ for all $k$, then $a=\max I_{f}(k) p_{\max }^{-2} \log \left(1 / p_{\max }\right)^{-2} \leq m^{-1 / 3}$, say, and Corollary 3 gives (3).

An alternative approach to proving results such as Lemma 1 is to adapt Talagrand's argument in [9] (see [3]); it may also be possible to use the approach of Rossignol [7]. His Corollary 3.1 does not seem to be strong enough as it is, however.

\section{References}

1. Bollobás, B., Riordan, O.: The critical probability for random Voronoi percolation in the plane is 1/2. Probability Theory and Related Fields 136, 417-468 (2006)

2. Bollobás, B., Riordan, O.: Percolation on random Johnson-Mehl tessellations and related models. Probability Theory and Related Fields 140, 319-343 (2008)

3. Bollobás, B., Riordan, O.: arXiv:0905.1275 (2009)

4. Bourgain, J., Kahn, J., Kalai, G., Katznelson, Y., Linial, N.: The influence of variables in product spaces. Israel J. Math. 77, 55-64 (1992)

5. Friedgut, E., Kalai, G.: Every monotone graph property has a sharp threshold. Proc. Amer. Math. Soc. 124, 2993-3002 (1996)

6. Kahn, J., Kalai, G., Linial, N.: The influence of variables on boolean functions. In: Proceedings of 29-th Annual Symposium on Foundations of Computer Science, pp. 68-80. Computer Society Press, UDS (1988)

7. Rossignol, R.: Threshold phenomena on product spaces: BKKKL revisited (once more). Electron. Commun. Probab. 13, 35-44 (2008)

8. Russo, L.: An approximate zero-one law. Z. Wahrscheinlichkeitstheorie Und Verw. Gebiete 61, 129139 (1982)

9. Talagrand, M.: On Russo's approximate zero-one law. Ann. Probab. 22, 1576-1587 (1994) 